\title{
Critical times: how has ecological research responded over the past 35
} years?

\author{
JONATHAN D. MAJER
}

Curtin Institute of Biodiversity and Climate, Curtin University, PO Box U 1987, Perth, WA 6845, Australia

\section{INTRODUCTION}

\author{
Woo ah mercy, mercy me \\ Ah, things ain't what they used to be \\ No, no, where did all the blue skies go \\ Poison is the wind that goes \\ From the north and south and east \\ Woo mercy, mercy me. \\ Lyrics Marvin P. Gaye \\ (C) EMI Music Publishing
}

So sang Marvin Gaye in his early 1970's Ecology Song, shortly before our fore-runner journal, Australian Journal of Ecology, commenced publication. Having spent 40 years researching insects in largely pristine habitats, I cannot help noticing that the majority of ecologists I meet are increasingly directing their research towards the numerous declines in the quality of our environment that the ever-expanding human population has caused. Looking at my own colleagues, I see people looking at what level of toxin entering a waterway can be tolerated by the resident fish, how successful we can be at restoring a destroyed ecosystem, how much ground water we can extract before various plant species can no longer transpire, how our ecosystems respond to invasive plants and animals, what happens to our diverse flora as the southwest of Western Australia dries out, and the list goes on. Depressing in some ways, but hopefully leading to some positive solutions!

To examine how ecological research in Australia has changed during my career, papers in each volume of Australian Journal of Ecology/Austral Ecology from volume 1 (1976) to volume 35 (2010) were categorized as being primarily directed towards species or 
ecosystems in relatively undisturbed conditions, or as addressing the consequences of a range of human-induced alterations of the environment. The categories adopted were: basic ecology; prescribed burning and managed fire; introduced species; agricultural and forestry pests; threatened species; habitat loss and fragmentation; replaced ecosystems (e.g., agriculture and plantations); miscellaneous threats (e.g., tourism, harvesting, pollution); restoration; and, climate change. Some papers traversed more than one category, in which case they were placed within the one that formed the major thrust of the paper. Book reviews, corrigenda, and thesis reviews were excluded from the counts.

Figure 1a indicates that the number of papers per year has grown steadily, whereas the percentage of papers devoted to basic ecology has progressively declined, dropping from around $80 \%$ in the first 6 years to less than $50 \%$ by 2010 (Figure 1b). This decline conforms with a linear model, with an $\mathrm{R}^{2}$ value of 0.42 and a significance of $p<0.001$ using two-tailed criteria.

The applied ecological papers are graphed in 5-year bands, as there is considerable variation in output between individual years. A number of trends are evident from these graphs. Firstly, papers concerning prescribed burning or managed fires (Fig. 2a) and those concerning replaced ecosystems, such as agricultural or forestry concerns (Fig. 2f), show no consistent trend over time, but rather oscillate in relation to current interests during the various periods.

Papers concerning the increasing threat of introduced species (Fig. 2b), miscellaneous threats ranging from deliberate or inadvertent exploitation of species or to pollution (Fig. $2 \mathrm{~g}$ ), and the loss or fragmentation of habitat (Fig. 2e), all of which are intimately linked with the conservation status of species (Fig. 2d), increased in numbers during the life of the journal. However, papers in the last mentioned category only became prevalent during the latter period of the journal's publication.

Restoration research exhibited a unimodal pattern, with a peak during the 1980's-90's (Fig. 2h). The upsurge in research probably tracks the increased pressure on companies to rehabilitate their land to high standards and also on the introduction of Landcare initiatives. Possible reasons for the subsequent decline are discussed later. Papers concerning pests, including the pathogen Phytophthora, have declined from an initial high level, and have remained low throughout the remaining period of the journal (Fig. 2c). 
The final category is global warming, papers on which are only starting to emerge in significant quantities during the past decade (Fig. 2i). Undoubtedly, this trend will continue upwards.

\section{DISCUSSION}

Even allowing for the inevitable biases or weaknesses of assigning papers to categories, and also the fact that metamorphosis to Austral Ecology opened up the journal to papers from throughout the southern hemisphere, clear trends in ecological research are evident. The progressive decline in the proportion of basic ecological research papers that are published is all the more surprising, since our parent Society introduced a second journal, Ecological Management and Restoration in 2000. Admittedly the content was supposed to be short, and directed towards managers, but it is likely that this would have directed some potential papers of an applied nature away from Austral Ecology. These trends should also be considered against a backdrop of external influencing factors. One is the upsurge in competing international journals, such as Ecology Letters, to which authors might be tempted to submit more theoretical papers in order to achieve higher coverage and citations and, currently, a higher Excellence in Research for Australia (ERA) ranking score. This is an Australian Government initiative which assesses national universities by, amongst other metrics, their publications, the quality of the journals in which they are published, and by the citation rates. Another is the establishment of more specialised journals, such as Restoration Ecology, that might attract articles which could otherwise be submitted to Austral Ecology. This could well be the reason for the decline in restoration-orientated papers, as Australian authors frequently contribute to this journal. It could also explain why few papers have been published on pest organisms in Austral Ecology in recent times, as there are now numerous journals concerned with such issues, such as the Australian journal, Crop and Pasture Science. Another possibility is that the research performed on pests is currently less ecological in nature or does not involve novel ecological findings (e.g., impact of GM crops, breeding for resistance), and is hence less suited for Austral Ecology.

Another influential driver is the ready availability of funds from companies and agencies concerned with resource development. Inevitably, this results in research that is directed towards restoration and environmental assessment. This pressure extends to governmentfunded research, where the success rate of applications for industry-associated Australian Research Council (ARC) Linkage grants is double that for ARC Discovery grants. As with non-government funding, this tends to result in mission-orientated, applied research. 
What is evident, however, is that ecologists are increasingly responding to threatening processes, including introduced species, loss or fragmentation of ecosystems, and exploitation of species, among others. As well as reflecting a concern by many ecologists for current environmental problems, this also results from the targeting of research funds from conservation agencies and from government sources, such as the ARC. One of the current National Priority Areas of the ARC is 'An environmentally sustainable Australia', funding from which may well be predisposed towards applied ecological projects. Naturally, the almost universal recognition that climate change has become a reality has resulted in considerable amounts of research now being focussed on this critical issue, in part because funding agencies are currently favouring applications which address this issue.

A further reason for performing research in disturbed ecosystems or under conditions of stress is that these tend to provide natural experiments. They provide ready-made manipulations and, often, the outcomes are magnified in comparison with experimental manipulations imposed on natural ecosystems. This can often result in extremely pronounced findings, possibly sufficient for publication in an ERA $A^{*}$ listed journal, in other words a journal that is deemed to be of the highest ranking at an international level for a particular discipline area! However, I suggest that such a reward is currently of lower importance to the long-term well-being of our world than is deriving a sound understanding of our ecosystems, their functioning, and the ecological requirements of their constituent species.

Encouraging as it may be that so much attention is being directed to environmental problems, there is still a role for basic ecology. The trends noted here suggest that research is largely being driven by politicians, bureaucrats and industry, rather than the converse. There is much to be learnt about the functioning of our ecosystems and of the species that occupy them, many of which are still new to science. It may well be that findings from a piece of basic ecological research provide an important breakthrough which enables us to resolve one of the pressing issues which are facing us today. Fortunately, the decline in proportion of papers devoted to basic ecology is offset by the overall increase in number of papers appearing in the journal. However, extrapolation of the relationship between the proportion of basic ecology articles against time suggests that most researchers will be addressing environmental issues rather than basic ecology in 30 years' time. Hopefully there will still be time to describe and understand our biota in order to ensure its survival and sustainability. If we don't, the future could be as bleak as the subsequent verses of the Ecology Song expound.

\section{ACKNOWLEDGEMENTS}


I thank Morgan Lythe for assisting with the data extraction from the journals and my friends and colleagues who bounced these ideas off me, namely Brian Heterick, Bernie Masters, Laco Mucina, Harry Recher and John Fox. 
(a)

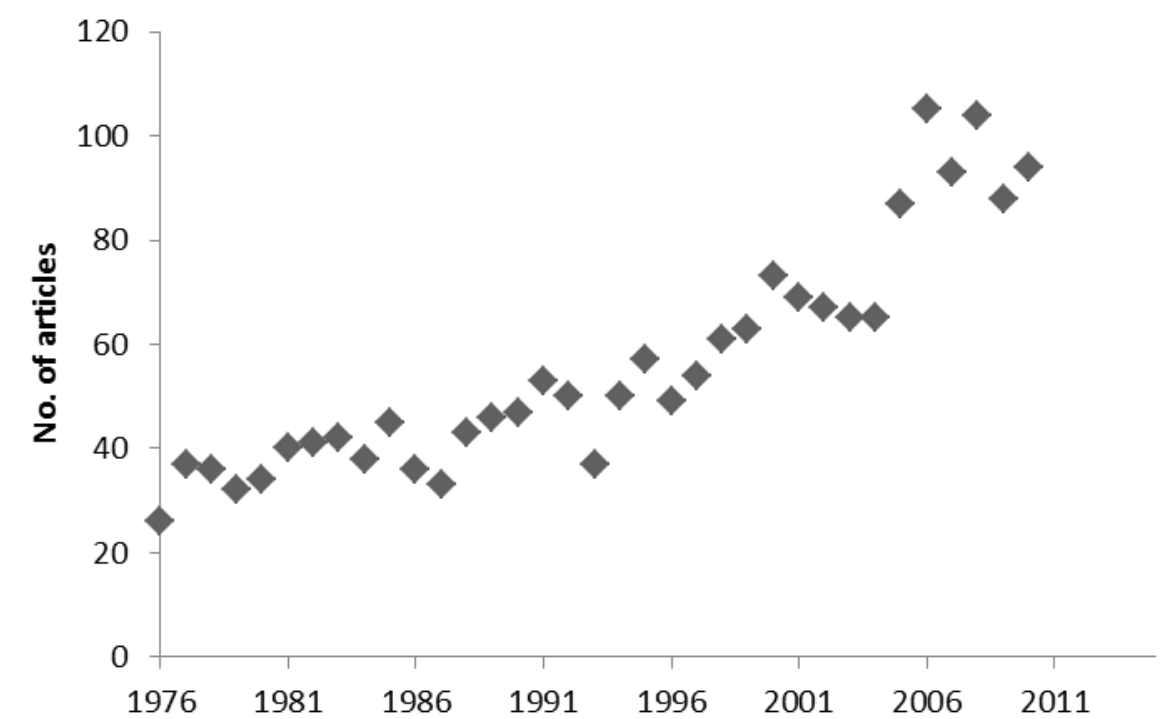

(b)

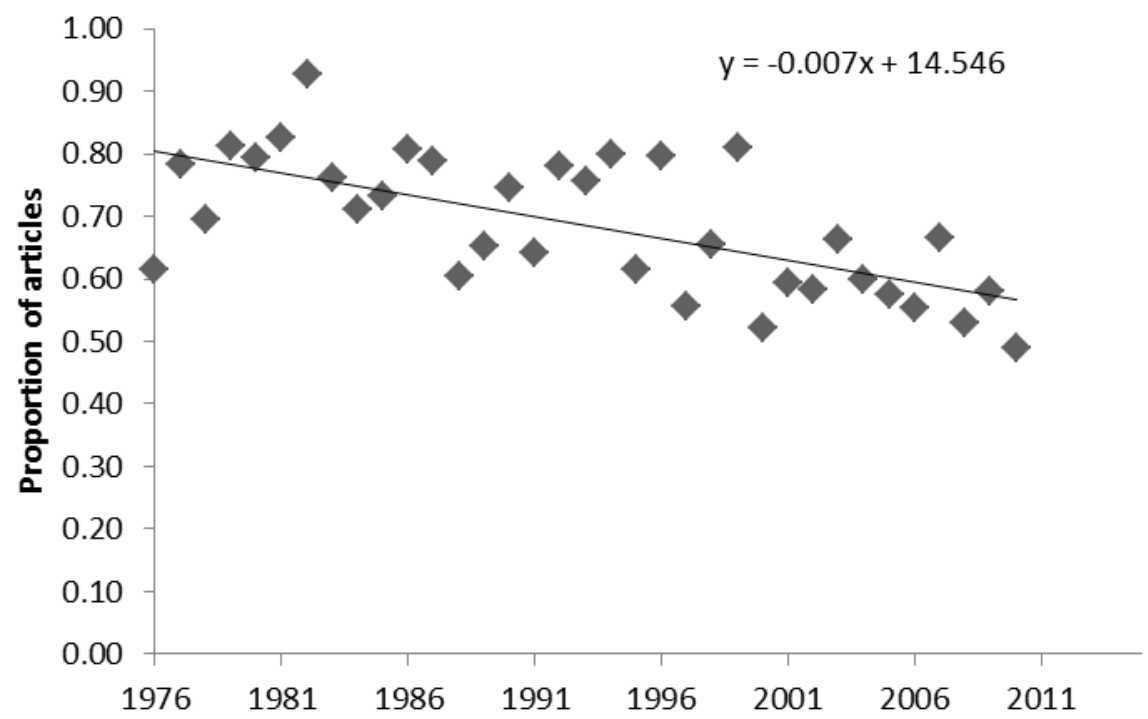

Fig. 1. Numbers of papers published per volume in Australian Journal of Ecology/Austral Ecology since its inception in 1976 until 2010 (a), and the proportion of papers in these volumes that are devoted to basic ecology (b). 


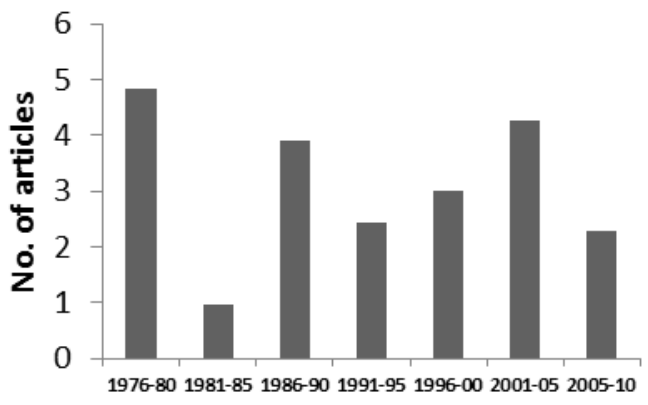

(c)

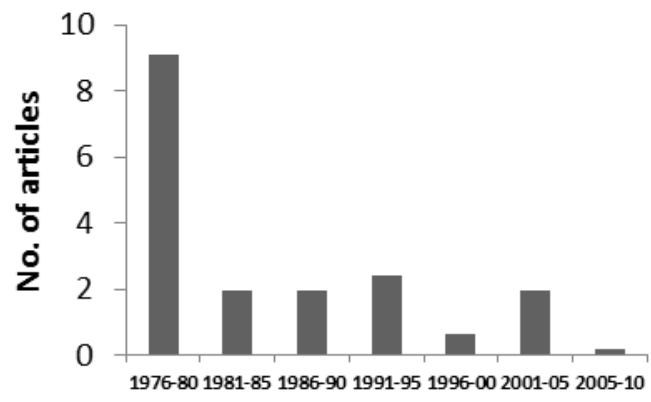

(e)

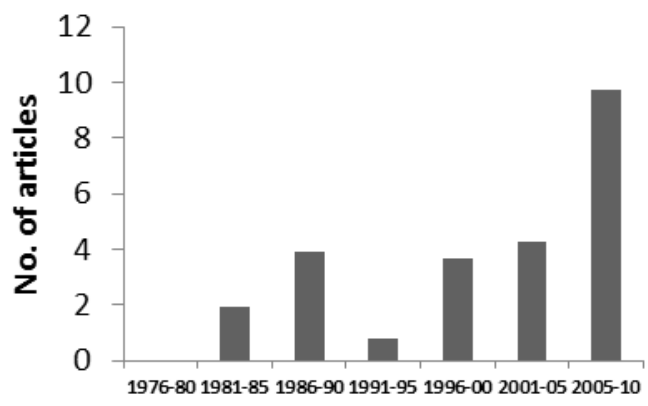

(g)

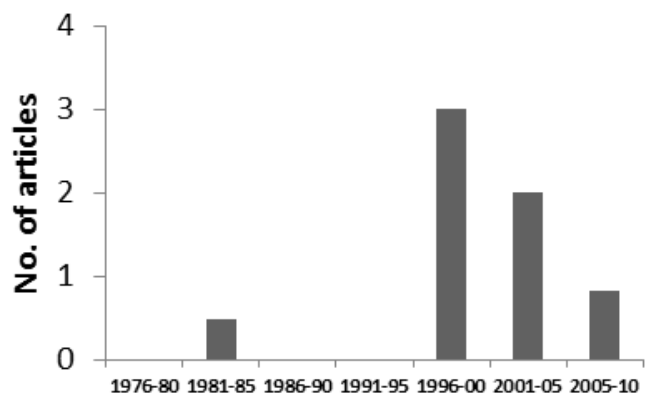

(i)

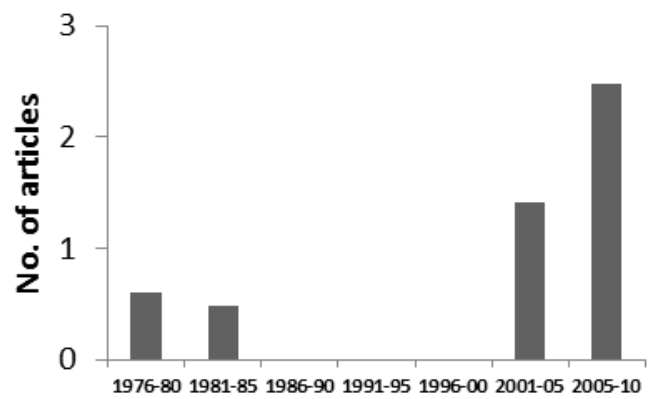

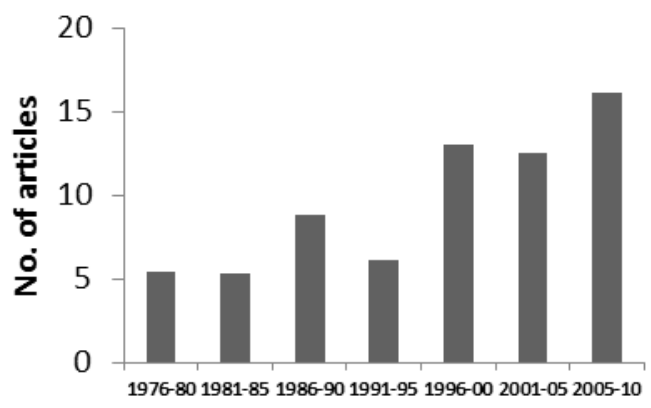

(d)

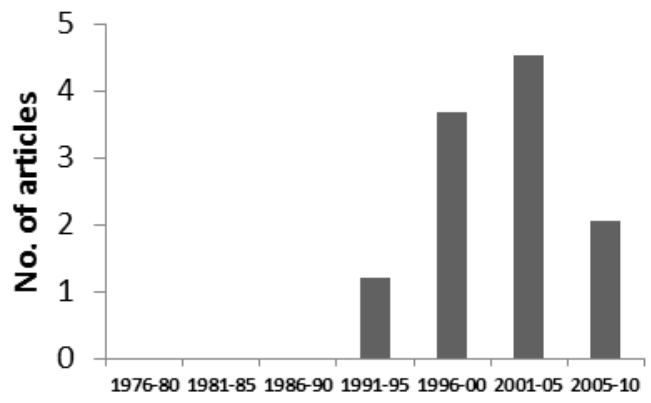

(f)

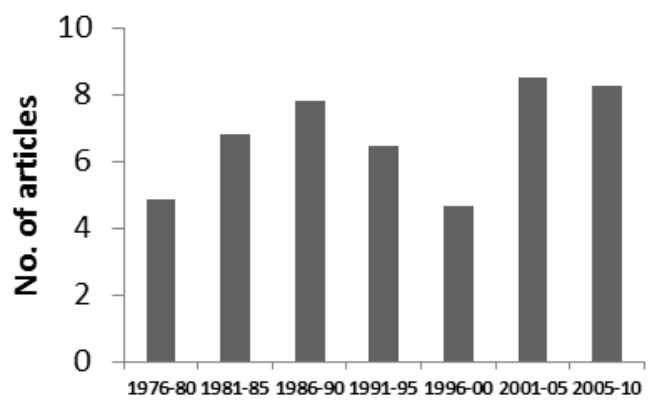

(h)

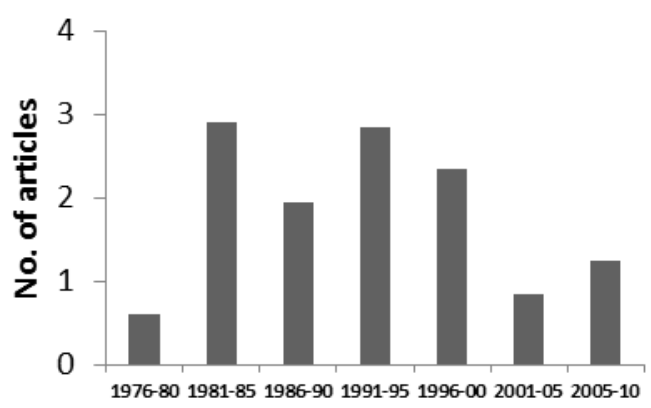

Fig. 2. Numbers of papers published per volume in Australian Journal of Ecology/Austral Ecology since its inception in 1976 until 2010, classified into: (a) prescribed burning and fire; (b) introduced species; (c) pests; (d) threatened species; (e) habitat loss and fragmentation; (f) replaced ecosystems; (g) miscellaneous threats; (h) restoration; and (i) climate change. 
